УДК 533.9

\title{
MAGNETIC ISLAND DYNAMICS UNDER EXTERNAL MAGNETIC PERTURBATION IN ROTATING RESISTIVE ITER PLASMA
}

\author{
N.V. Ivanov, A.M. Kakurin, S.V. Konovalov
}

NRC «Kurchatov Institute», Moscow, Russia

The modeling of the magnetic island evolution under resonant magnetic perturbations, RMP, in rotating ITER-like plasma from seed island initial generation, through appearance and development of the NTM till its locking and saturation is presented. The effects of plasma resistivity, viscosity, diamagnetic drift and the effect of the currents induced in the resistive vacuum vessel wall are taken into account. The calculations are carried out with the TEAR code based on the non-linear modified Rutherford model. The dependence of the tearing mode stability index on the angular position of the mode with respect to the RMP is taken into account. It results in deviations of the magnetic island rotation velocity from the velocity of the plasma layer in the vicinity of the rational magnetic surface. The magnetic island dynamics under RMP is analyzed for the modes originated at the $q=3 / 2$ and $q=2$ magnetic surfaces. The NTM behavior is discussed for cases of the seed island generation by different RMP sources including the coils for mitigation of the edge localized modes, ELMs.

Key words: tokamak, plasma, magnetic island, resonant magnetic perturbation, error field, neoclassical tearing mode.

\section{ДИНАМИКА МАГНИТНЫХ ОСТРОВОВ ВО ВРАЩАЮЩЕЙСЯ РЕЗИСТИВНОЙ ПЛАЗМЕ ИТЭР В ПРИСУТСТВИИ ВНЕШНИХ ВОЗМУЩЕНИЙ МАГНИТНОГО ПОЛЯ}

\author{
Н.В. Иванов, А.М. Какурин, С.В. Коновалов
}

НИЦ «Курчатовский институт», Москва, Россия

\begin{abstract}
Данная работа посвящена моделированию эволюции магнитных островов под действием резонансного магнитного возмущения (РMB) во вращающейся плазме на стадиях возбуждения исходного магнитного острова, возникновения и развития неоклассической тиринг-моды (НТМ), а также её захвата и насыщения. При моделировании учитываются эффекты резистивности и вязкости плазмы, диамагнитного дрейфа и влияния токов, индуцированных в резистивной стенке вакуумной камеры. Вычисления выполняются с помощью кода TEAR, базирующегося на нелинейной модифицированной модели Резерфорда. В расчётах учитывается зависимость индекса устойчивости тиринг-моды от углового положения моды по отношению к РМВ. Это приводит к отклонениям скорости вращения магнитных островов от скорости слоя плазмы в окрестности резонансной магнитной поверхности. В предлагаемой работе проводится анализ динамики магнитных островов на магнитных поверхностях $q=3 / 2$ и $q=2$ под действием РМВ. Обсуждаются динамика и возможности подавления НТМ в ИТЭР для случаев генерации исходных островов различными источниками PMB, включая обмотки для управления неустойчивостью ELM.
\end{abstract}

Ключевые слова: токамак, плазма, магнитный остров, резонансное магнитное возмущение, паразитное магнитное поле, неоклассическая тиринг-мода.

\section{INTRODUCTION}

The effects of externally applied resonant magnetic perturbation (RMP) or an error field on the magnetic islands attract considerable attention in the experimental and theoretical research of tokamak plasma. One of the main problems arising due to the RMP effect on the high-beta plasma is the development of magnetic islands acting as the seed islands for onset of the neoclassical tearing mode (NTM). In existing and next-step tokamaks including ITER, there are several sources of the magnetic perturbations: misalignments of the toroidal-field, central-solenoid and poloidal-field coils, joints and busbars in the magnetic system, blanket modules, irregularity in distribution of ferromagnetic inserts, NBI magnetic field reduction systems, bioshield, control coils for Edge Localized Modes etc. For ITER, a summation of the expected magnetic perturbations shows that in some regimes their amplitudes can exceed the level of $10^{-4}$ of the toroidal magnetic field.

The modeling of the magnetic island dynamics under RMP in rotating ITER-like plasma is presented in this paper. The paper is an extended version of the FEC-2012 report [1]. The calculations are carried out with the TEAR code [2,3] based on the two-fluid MHD approximation and modified non-linear Rutherford model [4]. In our model we allow for rotation of the magnetic perturbation relative to the resonant plasma layer. The magnetic island width much exceeds the width of the resistive layer in the conditions of the calculations. 


\section{COMPUTATIONAL MODEL}

Similar to [5-7], we use the generalized Ohm's law

$$
\partial \mathbf{B} / \partial t=\nabla \times(\mathbf{V} \times \mathbf{B}-\eta \mathbf{j})
$$

and the ion equation of motion

$$
\rho\left[\frac{\partial \mathbf{V}}{\partial t}+(\mathbf{V} \cdot \nabla) \mathbf{V}\right]=\mathbf{j} \times \mathbf{B}-\operatorname{div} \mathbf{\Pi}+\mathbf{f} .
$$

In these equations $\eta$ is the plasma resistivity, $\rho$ is the ion mass density, $\Pi$ is the viscous stress tensor given by Braginskii [8], $\mathbf{f}$ is the force not included in the first and second terms in the right-hand side of (2), $\mathbf{j}=\nabla \times \mathbf{B} / \mu_{0}, \mu_{0}$ is the magnetic permeability of vacuum. In this paper we consider a large aspect ratio tokamak with plasma major radius, $R$, and minor radius, $a$, in cylindrical approximation. The standard cylindrical polar coordinates $(r, \theta, z=\varphi R)$ are used. The plasma is assumed to be periodic in the «toroidal» $z$-direction with periodicity length $2 \pi R$. The magnetic field

$$
\mathbf{B}=B_{\theta 0} \mathbf{e}_{\theta}+B_{\varphi 0} \mathbf{e}_{\varphi}+\nabla \psi \times \mathbf{e}_{\varphi}
$$

includes the equilibrium part denoted by the subscript 0 and the perturbation expressed by the time dependent helical flux function

$$
\psi=\psi_{\mathrm{C}}(r, t) \cos (m \theta-n \varphi)+\psi_{\mathrm{S}}(r, t) \sin (m \theta-n \varphi) .
$$

In (4), $m$ and $n$ are the poloidal and toroidal mode numbers respectively. The space phase of the magnetic perturbation is defined as $\Phi(t)=\arctan \left(\psi_{\mathrm{S}} / \psi_{\mathrm{C}}\right)$ and the instantaneous value of the mode frequency is $\Omega(t)=d \Phi / d t$. The equation for the tearing mode evolution in time follows from (1):

$$
\frac{\partial \psi}{\partial t}+\mathbf{V} \nabla \psi=\frac{\eta}{\mu_{0}} \nabla^{2} \psi
$$

In the used model we assume that the force $\mathbf{f}$ does not depend on plasma rotation velocity and on the development of the tearing mode. In equation (2), we consider the axisymmetric velocities of the poloidal and toroidal plasma rotation and omit the convective plasma velocity:

$$
\mathbf{V}=V_{\theta}(r, t) \mathbf{e}_{\theta}+V_{\varphi}(r, t) \mathbf{e}_{\varphi} .
$$

In the absence of the tearing mode and RMP, the intrinsic plasma rotation velocity is

$$
\mathbf{V}_{0}=V_{\theta 0}(r) \mathbf{e}_{\theta}+V_{\varphi 0}(r) \mathbf{e}_{\varphi} .
$$

In the viscous term we take into account the radial transport of the poloidal and toroidal components of plasma momentum. The poloidal and toroidal components of the equation (2) give:

$$
\begin{gathered}
\rho \frac{\partial\left(V_{\theta}-V_{\theta 0}\right)}{\partial t}=f_{\mathrm{EM} \theta}+\mu_{\theta}\left\{\frac{1}{r} \frac{\partial}{\partial r}\left[r \frac{\partial\left(V_{\theta}-V_{\theta 0}\right)}{\partial r}\right]-\frac{V_{\theta}-V_{\theta 0}}{r^{2}}\right\} ; \\
\rho \frac{\partial\left(V_{\varphi}-V_{\varphi 0}\right)}{\partial t}=f_{\mathrm{EM} \varphi}+\frac{\mu_{\varphi}}{r} \frac{\partial}{\partial r}\left[r \frac{\partial\left(V_{\varphi}-V_{\varphi 0}\right)}{\partial r}\right] .
\end{gathered}
$$

In (8), (9), $f_{\mathrm{EM} \theta}$ and $f_{\mathrm{EM} \varphi}$ are the poloidal and toroidal components of the electromagnetic force $\mathbf{j} \times \mathbf{B}-\mathbf{j}_{0} \times \mathbf{B}_{0}$, where $\mathbf{j}_{0}=\nabla \times \mathbf{B}_{0} / \mu_{0} ; \mu_{\theta}$ and $\mu_{\varphi}$ are the poloidal and toroidal components of plasma viscosity. In this paper, we disregard the radial dependence of the plasma viscosity.

The TEAR code is based on the solution of the coupled diffusion-type equations for the helical flux function (5) and for the plasma rotation velocity (8), (9). The equation (5) describes the dynamics of the tearing mode. The second term in the left-hand side of this equation depends on the plasma rotation. In sequence, this rotation is affected by the electromagnetic forces in (8), (9) depending on the tearing mode magnetic field and RMP. Numerically, the diffusion-type equations for the helical flux function and for the plasma rotation velocity 
are treated in a similar way. We consider the resonant plasma layer in the vicinity of the resonant magnetic surface with radius $r_{\mathrm{S}}$, where safety factor is $q\left(r_{\mathrm{S}}\right)=\frac{r_{\mathrm{S}} B_{\varphi 0}}{R B_{\theta 0}}=\frac{m}{n}$. We assume that the width of the resonant layer matches the width of the magnetic island:

$$
W=\left.4 \sqrt{\frac{q \sqrt{\psi_{\mathrm{C}}^{2}+\psi_{\mathrm{S}}^{2}}}{B_{\theta 0}|d q / d r|}}\right|_{r_{\mathrm{s}}} .
$$

In our model we use the conventional constant- $\psi$ approximation inside the resonant layer. We also assume that the plasma momentum transport is high and consequently the plasma velocity is homogeneous inside the resonant layer.

Equations for helical flux function. The equation (5) can be re-written as separate equations for the cosine and sine harmonics introduced by (4):

$$
\begin{aligned}
& \frac{\partial \psi_{\mathrm{C}}}{\partial t}+\left(\frac{m V_{\theta}}{r}-\frac{n V_{\varphi}}{R}\right) \psi_{\mathrm{S}}=\frac{\eta}{\mu_{0}} \nabla^{2} \psi_{\mathrm{C}} ; \\
& \frac{\partial \psi_{\mathrm{S}}}{\partial t}-\left(\frac{m V_{\theta}}{r}-\frac{n V_{\varphi}}{R}\right) \psi_{\mathrm{C}}=\frac{\eta}{\mu_{0}} \nabla^{2} \psi_{\mathrm{S}} .
\end{aligned}
$$

Similar to [2], the equations (11) and (12) are integrated across the resonant layer $r_{\mathrm{S}}-W / 2<r<r_{\mathrm{S}}+W / 2$. After the integration in assumption that $W<<a$, the equations (11), (12) give two equations for the time evolution of helical flux function cosine and sine harmonics at $r=r_{\mathrm{S}}$ :

$$
\begin{aligned}
& \frac{\partial \psi_{\mathrm{C}}}{\partial t}=\left.\frac{\eta}{\mu_{0} W} \frac{\partial \psi_{\mathrm{C}}}{\partial r}\right|_{r_{\mathrm{S}}-W / 2} ^{r_{\mathrm{S}}+W / 2}-\left(\frac{m V_{\theta}}{r}-\frac{n V_{\varphi}}{R}\right) \psi_{\mathrm{S}} \\
& \frac{\partial \psi_{\mathrm{S}}}{\partial t}=\left.\frac{\eta}{\mu_{0} W} \frac{\partial \psi_{\mathrm{S}}}{\partial r}\right|_{r_{\mathrm{S}}-W / 2} ^{r_{\mathrm{S}}+W / 2}+\left(\frac{m V_{\theta}}{r}-\frac{n V_{\varphi}}{R}\right) \psi_{\mathrm{C}}
\end{aligned}
$$

For these equations we apply the notations:

$$
\begin{gathered}
\Delta_{\mathrm{C}}^{\prime}(W)=\left.\frac{1}{\psi_{\mathrm{C}}\left(r_{\mathrm{S}}\right)} \frac{\partial \psi_{\mathrm{C}}}{\partial r}\right|_{r_{\mathrm{S}}-W / 2} ^{r_{\mathrm{S}}+W / 2} ; \\
\Delta_{\mathrm{S}}^{\prime}(W)=\left.\frac{1}{\psi_{\mathrm{S}}\left(r_{\mathrm{S}}\right)} \frac{\partial \psi_{\mathrm{S}}}{\partial r}\right|_{r_{\mathrm{S}}-W / 2} ^{r_{\mathrm{S}}+W / 2},
\end{gathered}
$$

that are the cosine and sine components of the tearing mode stability index. In general case, the stability index components $\Delta_{\mathrm{C}}^{\prime}$ and $\Delta_{\mathrm{S}}^{\prime}$ are found separately for both the cosine and sine space harmonics of the flux function similar to $[2,3,5]$. Without RMP, $\Delta_{\mathrm{C}}^{\prime}=\Delta_{\mathrm{S}}^{\prime}$, one can obtain from (10), (13) - (16) the conventional Rutherford equation for the time variation of the magnetic island width.

In the model used for the TEAR code numerical calculations, we apply the factor $\pi$ at the first terms in the right-hand side of equations (13), (14) in accordance with [2, 9]. In this model we also supplement the equations (13), (14) with the neoclassical term and the term for the electron diamagnetic drift:

$$
\begin{aligned}
& \frac{d \psi_{\mathrm{C}}}{d t}=\frac{\pi a^{2} \omega_{\mathrm{R}}}{W}\left(\Delta_{\mathrm{C}}^{\prime}+\Delta_{\mathrm{NC}}^{\prime}\right) \psi_{\mathrm{C}}-\Omega_{\mathrm{nat}} \psi_{\mathrm{S}} ; \\
& \frac{d \psi_{\mathrm{S}}}{d t}=\frac{\pi a^{2} \omega_{\mathrm{R}}}{W}\left(\Delta_{\mathrm{S}}^{\prime}+\Delta_{\mathrm{NC}}^{\prime}\right) \psi_{\mathrm{S}}+\Omega_{\mathrm{nat}} \psi_{\mathrm{C}},
\end{aligned}
$$

where $\omega_{\mathrm{R}}=\eta / \mu_{0} a^{2}$ is the inverse resistive time; $\Delta_{\mathrm{NC}}^{\prime}(W)$ is the neoclassical term [10-12]; $\Omega_{\text {nat }}=\left.\left(m V_{\theta} / r_{\mathrm{S}}-n V_{\varphi} / R-\Omega_{e^{*}}\right)\right|_{r_{\mathrm{S}}}$ is the natural frequency depending on the poloidal, $V_{\theta}$, and toroidal, $V_{\varphi}$, rotation 
velocities of the resonant plasma layer as well as on the frequency of the electron diamagnetic drift, $\Omega_{e^{*}}$, [5, 6 , 13-16]. In the absence of the tearing mode and RMP, the natural frequency is equal to its intrinsic value $\Omega_{\text {nat } 0}=\left.\left(m V_{\theta 0} / r_{\mathrm{S}}-n V_{\varphi 0} / R-\Omega_{e^{*}}\right)\right|_{r_{\mathrm{S}}}$ that is considered as an external parameter in our calculations.

According to the standard approach in the tearing mode theory, the electromagnetic force is neglected in all plasma volume except the resonant layer. The condition for force balance $\mathbf{j} \times \mathbf{B}-\mathbf{j}_{0} \times \mathbf{B}_{0}=0$ gives the equations

$$
\begin{aligned}
& \frac{1}{r} \frac{\partial}{\partial r}\left(r \frac{\partial \psi_{\mathrm{C}}}{\partial r}\right)-\left(\frac{m^{2}}{r^{2}}+\frac{\mu_{0}}{B_{\theta 0}} \frac{d j_{\varphi 0} / d r}{1-n q / m}\right) \psi_{\mathrm{C}}+\mu_{0} \sum_{k} i_{k \mathrm{C}} \delta\left(r-r_{k}\right)=0 ; \\
& \frac{1}{r} \frac{\partial}{\partial r}\left(r \frac{\partial \psi_{\mathrm{S}}}{\partial r}\right)-\left(\frac{m^{2}}{r^{2}}+\frac{\mu_{0}}{B_{\theta 0}} \frac{d j_{\varphi 0} / d r}{1-n q / m}\right) \psi_{\mathrm{S}}+\mu_{0} \sum_{k} i_{k \mathrm{~S}} \delta\left(r-r_{k}\right)=0,
\end{aligned}
$$

which are used for the calculation of the radial distribution of the cosine and sine components of the helical flux function in areas $0 \leq r \leq r_{\mathrm{S}}-W / 2$ and $r_{\mathrm{S}}+W / 2 \leq r \leq b$, where $b$ is the effective radius of the perfectly conducting outer wall. The solutions of (19), (20) satisfy the boundary conditions $\psi_{\mathrm{C}, \mathrm{S}}\left(r_{\mathrm{S}}-W / 2\right)=\psi_{\mathrm{C}, \mathrm{S}}\left(r_{\mathrm{S}}+W / 2\right)=\psi_{\mathrm{C}, \mathrm{S}}\left(r_{\mathrm{S}}\right)$ and $\psi_{\mathrm{C}, \mathrm{S}}(0)=\psi_{\mathrm{C}, \mathrm{S}}(b)=0$. In this paper we assume that $b \rightarrow \infty$. In (19) and (20), $j_{\varphi 0}(r)$ is the unperturbed part of the plasma current density. In the terms with delta function $\delta\left(r-r_{k}\right)$ in (19), (20), the $i_{k \mathrm{C}}$ and $i_{k \mathrm{~S}}$ are the cosine and sine components of surface densities of the external helical currents at radii $r=r_{k} \geq a$ :

$$
i_{k}=i_{k \mathrm{C}}(t) \cos (m \theta-n \varphi)+i_{k \mathrm{~S}}(t) \sin (m \theta-n \varphi) .
$$

For ITER simulation, we consider three surface currents outside the plasma, including the current, producing the external RMP and two currents with the surface densities

$$
i_{\mathrm{C}, \mathrm{s}}=-\sigma h \frac{d \psi_{\mathrm{C}, \mathrm{s}}}{d t},
$$

generated in two ITER resistive vacuum vessel walls at different effective radii $r=r_{\mathrm{vv} 1}$ and $r=r_{\mathrm{vv} 2}$ due to the magnetic flux variations in time. In the formula (22), $h$ is the effective thickness of the vacuum vessel wall and $\sigma$ is the wall electrical conductivity.

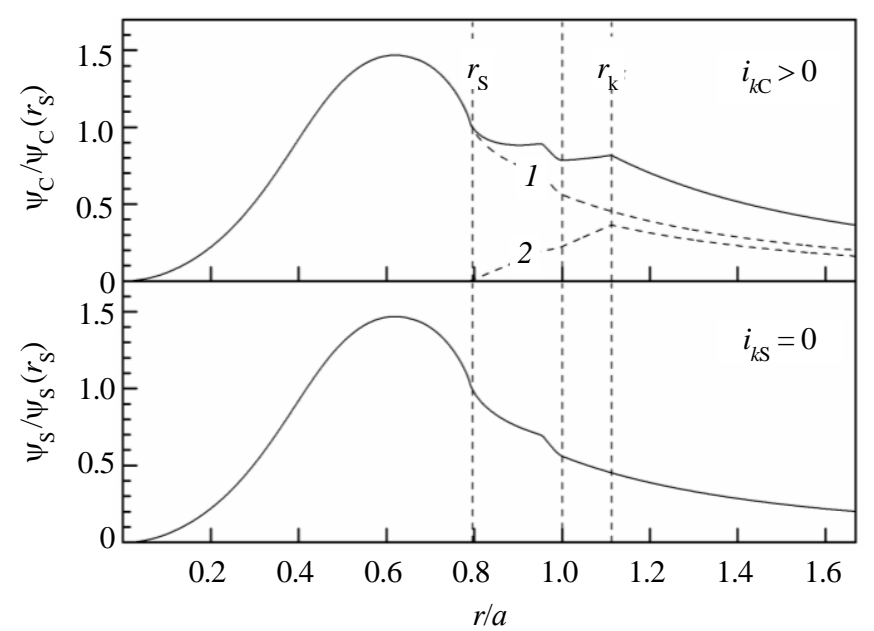

Fig. 1. The solutions of equations (19), (20) for the case of $i_{k \mathrm{C}} \neq 0, i_{k \mathrm{~S}}=0$ are shown with solid curves. Dashed curves in the upper panel represent the general solution of homogeneous equation (19) with $i_{k \mathrm{C}}=0$ (dashed curve 1) and particular solution of the non-homogeneous equation (19) with $i_{k \mathrm{C}} \neq 0$ (dashed curve 2 )
Each solution of the equations (19), (20) consists of the general solution of the corresponding homogeneous equation with $i_{k \mathrm{C}, \mathrm{s}}=0$ and particular solution of the non-homogeneous equation that depends on the cosine and sine components of the external helical current $i_{k \mathrm{C}, \mathrm{s}}$. An example of these solutions is shown for illustration in Fig. 1.

The stability index cosine and sine components found from the solution of (19), (20) consist of the common symmetric part, $\Delta_{0}^{\prime}$, which is calculated without the RMP $\left(i_{k \mathrm{C}}=i_{k \mathrm{~S}}=0\right)$, and the additions arising due to the terms with the asymmetric external helical currents. The instantaneous value of the mode frequency obtained from (17), (18) differs from the natural frequency due to the asymmetric effect of the RMP on the tearing mode stability index: 


$$
\Omega(t)=\frac{d}{d t}\left(\arctan \frac{\psi_{\mathrm{S}}}{\psi_{\mathrm{C}}}\right)=\Omega_{\mathrm{nat}}+\frac{\pi a^{2} \omega_{\mathrm{R}}}{W}\left(\Delta_{\mathrm{S}}^{\prime}-\Delta_{\mathrm{C}}^{\prime}\right) \frac{\psi_{\mathrm{C}} \psi_{\mathrm{S}}}{\psi_{\mathrm{C}}^{2}+\psi_{\mathrm{S}}^{2}} .
$$

Equations for resonant layer rotation. The rotation velocities of the resonant plasma layer are obtained from equations (8), (9). We integrate the equations (8), (9) over the resonant layer under the assumption that the plasma velocity is homogeneous inside this layer:

$$
\begin{aligned}
& \frac{I_{\theta}}{r_{\mathrm{S}}} \frac{\partial\left(V_{\theta}-V_{\theta 0}\right)}{\partial t}=T_{\mathrm{EM} \theta}+T_{\mathrm{V} \theta} ; \\
& \frac{I_{\varphi}}{R} \frac{\partial\left(V_{\varphi}-V_{\varphi 0}\right)}{\partial t}=T_{\mathrm{EM} \varphi}+T_{\mathrm{V} \varphi},
\end{aligned}
$$

where $I_{\theta}$ and $I_{\varphi}$ are the poloidal and toroidal moments of inertia of the plasma resonant layer, $T_{\mathrm{EM} \theta}$ and $T_{\mathrm{EM} \varphi}$ are the electromagnetic torques, $T_{\mathrm{V} \theta}$ and $T_{\mathrm{V} \varphi}$ are the viscous friction torques. The electromagnetic torque is calculated as the reaction to the total torque applied to the conductors with the external helical currents from the magnetic field perturbation caused by the tearing mode. The viscous torques are:

$$
\begin{gathered}
T_{\mathrm{V} \theta}=4 \pi^{2} r_{\mathrm{S}}^{2} R \mu_{\theta} \Delta_{\mathrm{V} \theta}^{\prime}\left(V_{\theta}-V_{\theta 0}\right) ; \\
T_{\mathrm{V} \varphi}=4 \pi^{2} r_{\mathrm{S}} R^{2} \mu_{\varphi} \Delta_{\mathrm{V} \varphi}^{\prime}\left(V_{\varphi}-V_{\varphi 0}\right) .
\end{gathered}
$$

Here we introduce the factors:

$$
\begin{aligned}
& \Delta_{\mathrm{V} \theta}^{\prime}=\left.\frac{1}{V_{\theta}-V_{\theta 0}} \frac{\partial\left(V_{\theta}-V_{\theta 0}\right)}{\partial r}\right|_{r_{\mathrm{s}}-W / 2} ^{r_{s}+W / 2} ; \\
& \Delta_{\mathrm{V} \varphi}^{\prime}=\left.\frac{1}{V_{\varphi}-V_{\varphi 0}} \frac{\partial\left(V_{\varphi}-V_{\varphi 0}\right)}{\partial r}\right|_{r_{\mathrm{s}}-W / 2} ^{r_{\mathrm{s}}+W / 2},
\end{aligned}
$$

by analogy with the components of the tearing mode stability index. We call the factors (28) and (29) the poloidal and toroidal components of the viscous friction index, respectively. These components are obtained from the solutions of the equations (8), (9) in intervals $0 \leq r \leq r_{\mathrm{s}}-W / 2$ and $r_{\mathrm{s}}+W / 2 \leq r \leq a$ outside the resonant plasma layer. The boundary conditions for the equation (8) are:

$$
\begin{gathered}
\left.\left(V_{\theta}-V_{\theta 0}\right)\right|_{r=0}=0 ; \\
\left.\frac{V_{\theta}-V_{\theta 0}}{r}\right|_{r_{\mathrm{s}}-W / 2}=\left.\frac{V_{\theta}-V_{\theta 0}}{r}\right|_{r_{\mathrm{s}}+W / 2}=\left.\frac{V_{\theta}-V_{\theta 0}}{r}\right|_{r_{\mathrm{s}}}
\end{gathered}
$$

and

$$
\left.\left(V_{\theta}-V_{\theta 0}\right)\right|_{r=a}=0
$$

The boundary conditions for the equation (9) are:

$$
\begin{gathered}
\left.\frac{\partial\left(V_{\varphi}-V_{\varphi 0}\right)}{\partial r}\right|_{r=0}=0 ; \\
\left.\left(V_{\varphi}-V_{\varphi 0}\right)\right|_{r_{\mathrm{s}}-W / 2}=\left.\left(V_{\varphi}-V_{\varphi 0}\right)\right|_{r_{\mathrm{s}}+W / 2}=\left.\left(V_{\varphi}-V_{\varphi 0}\right)\right|_{r_{\mathrm{s}}}
\end{gathered}
$$

and

$$
\left.\left(V_{\varphi}-V_{\varphi 0}\right)\right|_{r=a}=0 .
$$


The boundary conditions at the borders of the resonant layer, $r=r_{\mathrm{s}} \pm W / 2$, depend on time and are obtained from the solutions of equations (24), (25).

\section{CONDITIONS AND RESULTS OF CALCULATIONS}

The calculations were performed for the plasma parameters of ITER inductive scenario with plasma current 15 MA (Scenario \# 2) [17]. The geometry of the two resistive vacuum vessel walls was specified as in [18]. The values of plasma viscosity components were chosen under the assumptions that the Prandtl number [19] can be of the order of or a few times exceeding unity and that the poloidal viscosity is much higher than the toroidal viscosity. Under these assumptions, the values of the toroidal and poloidal momentum radial diffusivity $\mu_{\varphi} / \rho=3 \mathrm{~m}^{2} \cdot \mathrm{s}^{-1}$ and $\mu_{\theta} / \rho=300 \mathrm{~m}^{2} \cdot \mathrm{s}^{-1}$ were used in the calculations.

According to [20], we supposed that the expected amplitudes of the poloidal RMP component at plasma edge for the $m=3, n=2$ and $m=2, n=1$ modes do not exceed $10^{-3} \mathrm{~T}$. The vacuum magnetic perturbations due to ELM control coils were calculated directly from Biot-Savart law as in [21] with account of ITER ELM coil geometry [22] and setting the currents in each of 27 ELM coils as

$$
I_{t, m, b}(\mathrm{kA})=90 \sin \left[4\left(2 \pi k / 9-30^{\circ}+\alpha_{t, m, b}\right)\right],
$$

where indices $t, m, b$ state for the top, middle and bottom rows of coils, $k$ is the coil number in the raw and phase shifts were taken $\alpha_{t, m b}=\left\{54^{\circ}, 0,-63^{\circ}\right\}$ respectively. Precalculated 2D-distributions of the perturbed poloidal fields on the resonant surfaces $q=\{3 / 2,2\}$ were expanded into double Fourier series and the cylindrical equivalents for dominant harmonics were determined. Then, the maximum expected amplitudes of the external helical currents, entering equations (19), (20), were calculated with use of the TEAR code. The maximum expected RMP amplitudes caused by the ELM control coils at plasma edge were found to be of $2 \cdot 10^{-3} \mathrm{~T}$ for the $m=6, n=4$ and $m=8, n=4$ modes. The amplitudes of the most dangerous components of the ELM coil perturbation with $m=3, n=2$ and $m=2, n=1$ were found to be less than $3 \cdot 10^{-5} \mathrm{~T}$.

Following [23], the neoclassical contribution into equations (17), (18) takes the form

$$
\Delta_{\mathrm{NC}}^{\prime}=r_{\mathrm{S}} \beta_{\mathrm{P}}\left(\Delta_{\mathrm{BS}}^{\prime} \frac{W}{W^{2}+W_{\mathrm{C}, \mathrm{BS}}^{2}}+\Delta_{\mathrm{MW}}^{\prime} \frac{1}{W+W_{\mathrm{C}, \mathrm{MW}}}\right),
$$

where the first term in the brackets corresponds to the bootstrap drive of the neoclassical tearing mode and the second one to the stabilizing magnetic curvature effect, $W_{\mathrm{C}, \mathrm{BS}}$ and $W_{\mathrm{C}, \mathrm{MW}}$ are the critical island widths [23] characterizing the NTM evolution in the small island limit. Numerical calculations of the neoclassical terms based on the algorithms outlined in [24] for the bootstrap drive and in [25] for the magnetic curvature effect were performed for the ITER Scenario \#2 reference data [17] resulting in $r_{\mathrm{S}} \beta_{\mathrm{P}} \Delta_{\mathrm{BS}}^{\prime}=0.2, \Delta_{\mathrm{MW}}^{\prime} \approx-0.1 \times \Delta_{\mathrm{BS}}^{\prime}$ at the $q=3 / 2$ surface and $\Delta_{\mathrm{MW}}^{\prime} \approx-0.2 \times \Delta_{\mathrm{BS}}^{\prime}$ at the $q=2 / 1$ surface. In the cylindrical approximation, the curvature effect is weaker compared to the bootstrap drive, $\Delta_{\mathrm{MW}}^{\prime} / \Delta_{\mathrm{BS}}^{\prime} \sim \varepsilon^{3 / 2}$. Due to the different dependence on $W$, the curvature effect can overcome the drive and completely stabilize the mode at small island width, $W \sim W_{\mathrm{C}}$. In the ITER case, theoretical estimation according to [23] predicts that $W_{\mathrm{C}, \mathrm{BS}}$ should be of the order of a few centimeters. This estimation well coincides with empirical findings characterizing $W_{\mathrm{C}, \mathrm{BS}}$ in terms of a few ion Larmor radii. In our present analysis we consider $1 \mathrm{~cm}<W_{\mathrm{C}, \mathrm{BS}}<6 \mathrm{~cm}$, setting $W_{\mathrm{C}, \mathrm{MW}}=0.4 \times W_{\mathrm{C}, \mathrm{BS}}$.

The radial distribution of the unperturbed plasma current density used in the calculations is shown in Fig. 2. In Fig. 3, one can see the dependences of the symmetric part of the stability index, $\Delta_{0}^{\prime}$, on the magnetic island width for the modes under consideration at the $q=3 / 2$ and $q=2$ magnetic surfaces. According to the calculations, only $m=2$, $n=1$ mode is originally unstable $\left(\Delta_{0}^{\prime}>0\right.$ ) for the chosen current profile. It is well known that the tearing mode stability strongly depends on the current profile. We illustrate it in Fig. 2 and Fig. 3 for the mode $m=2, n=1$. The small variation of the current profile shown with the dashed curve in Fig. 2 makes the mode stable $\left(\Delta_{0}^{\prime}<0\right)$ for arbitrary 


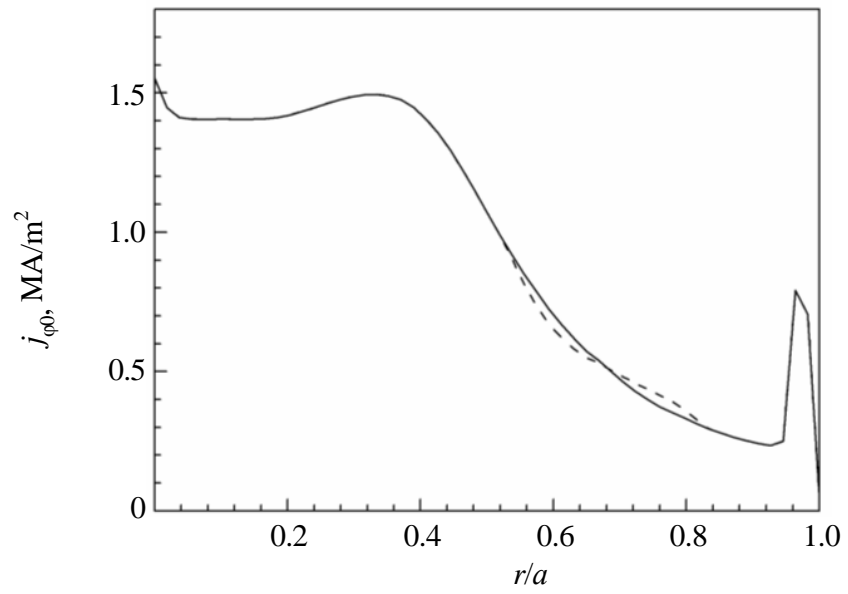

Fig. 2. Radial profile of the unperturbed plasma current density

island width (see dashed line in Fig. 3). It turns out that in presence of RMP the widths of magnetic islands do not strongly depend on the symmetric part of the stability index if this part is negative, $\Delta_{0}^{\prime}<0$. This is illustrated by Fig. 4 where the dependences of the magnetic island width on the artificial shift of the curves, $\Delta_{0}^{\prime}(W) \rightarrow \Delta_{0}^{\prime}(W)+C$, along the ordinate axis by $C$ scan are shown. The calculations presented in Fig. 4 were done for $\Omega_{\text {nato }} / 2 \pi=50 \mathrm{~Hz}$ and for the amplitude of the RMP poloidal component at plasma edge, $B_{\mathrm{RMP}}=2 \cdot 10^{-4} \mathrm{~T}$, the neoclassical effects were neglected.

The dependences of the width of the magnetic islands generated by RMP for different tearing mode harmonics on the amplitude of RMP (at $\Omega_{\text {nato }} / 2 \pi=50 \mathrm{~Hz}$ )

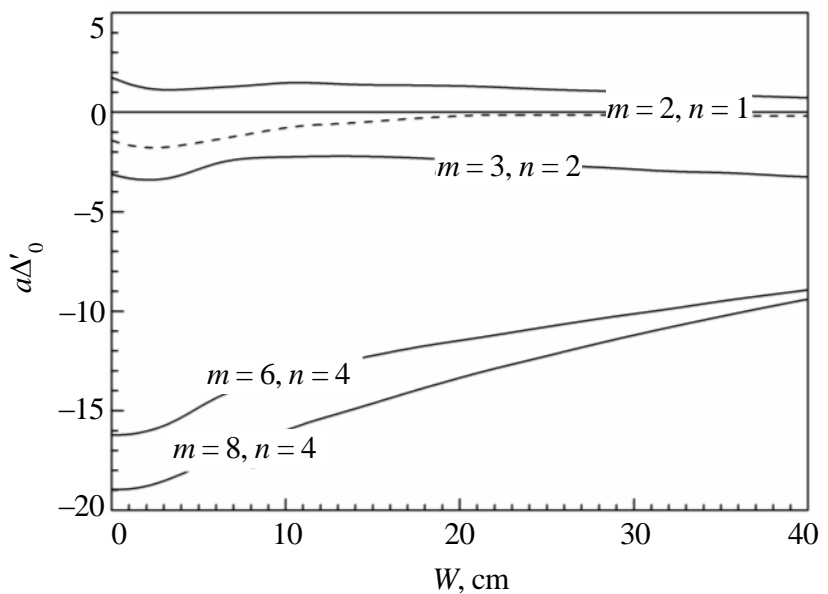

Fig. 3. Dependences of the symmetric part of the stability index on $W$ for different mode numbers

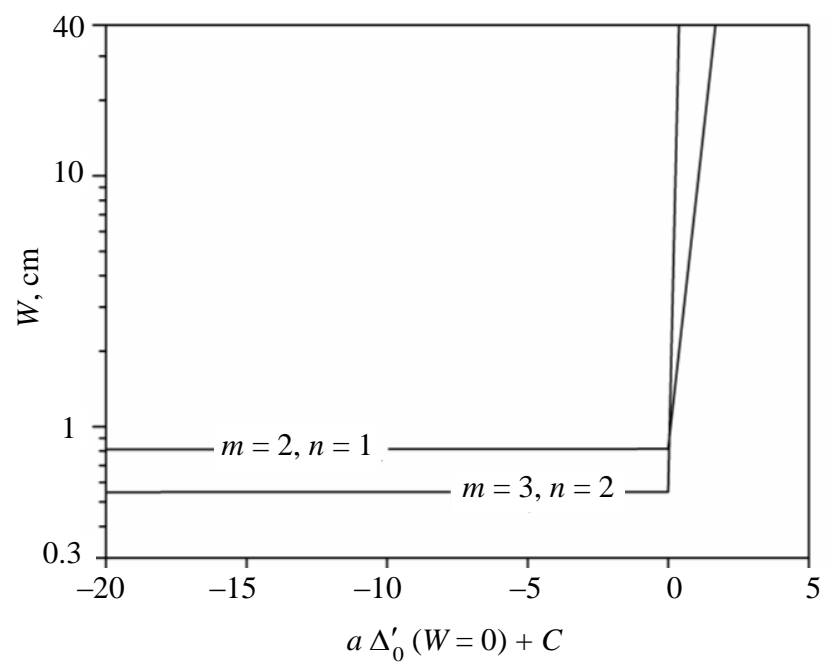

Fig. 4. Dependences of the magnetic island width on the symmetric part of the stability index and on the intrinsic frequency, $\Omega_{\text {nat } 0}$, (at maximum expected RMP amplitude for each mode) are shown in Fig. 5 and Fig. 6. To distinguish the RMP effect from the neoclassical effects, the neoclassical terms were omitted in calculations for these two figures. In calculations presented in Fig. 5 and Fig. 6, the magnetic islands produced by non-rotating RMP were locked $(\Omega=0)$ despite the natural frequency $\Omega_{\text {nat }} \neq 0$. Since a small correction of the current profile can make the tearing mode originally stable and the magnetic islands generated by RMP do not
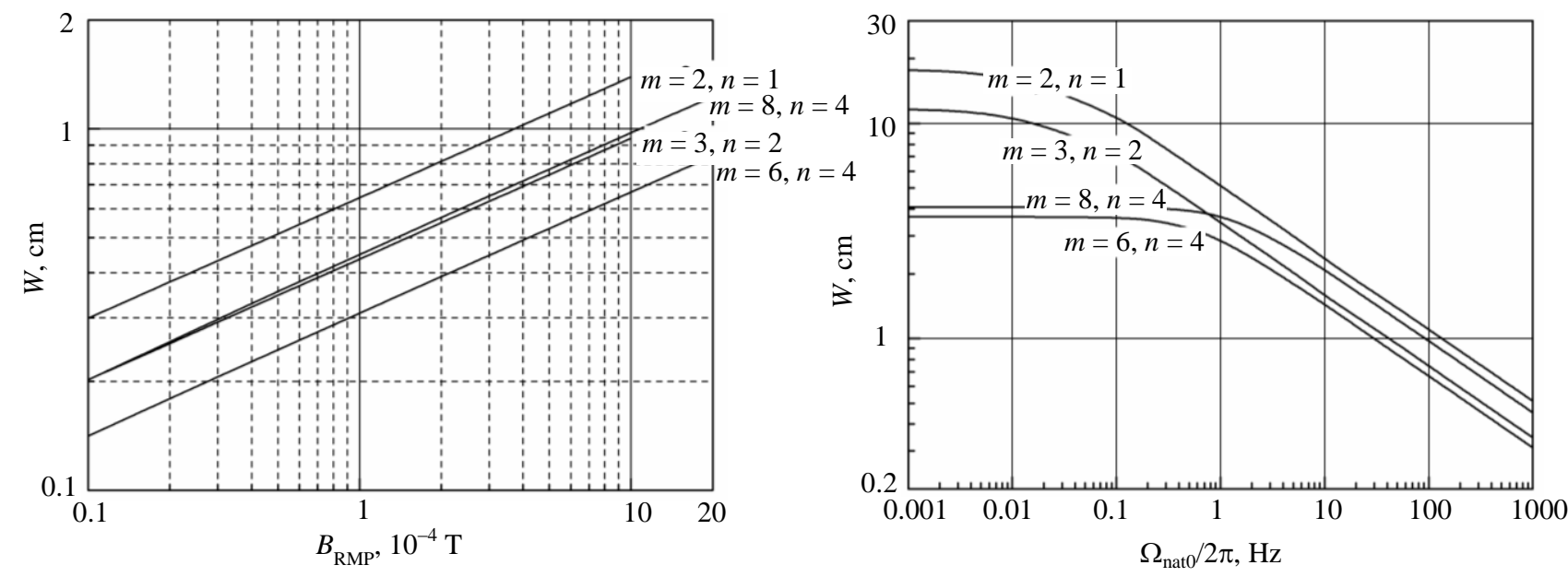

Fig. 5. Dependences of the magnetic island width on the RMP amplitude

Fig. 6. Dependences of the magnetic island width on the intrinsic natural frequency 
significantly depend on the negative $\Delta_{0}^{\prime}$, in the calculations presented in Fig. 5 and Fig. 6 a simple assumption that $\Delta_{0}^{\prime}<0$ was used for all mode numbers under consideration. The calculations for Fig. 5 and Fig. 6 were performed using current profiles shown in Fig. 2 with the dashed curve for the $m=2, n=1$ and with the solid curves for other modes. One can see that the magnetic island width values increase with the rise of the RMP amplitude and decrease with the rise of the intrinsic frequency. This character of the island width dependence on RMP and $\Omega_{\text {nat0 }}$ can be explained from the consideration of formula (23) in the conditions when $\Omega=0, \Omega_{\text {nat }} \approx \Omega_{\text {nat0 }}$ and the tearing mode stability index components are nearly proportional to the RMP.

The process of subsequent development of the NTM from RMP produced seed islands was examined in the series of calculations for a wide range of the mode numbers, rotation frequencies, $\Omega_{\text {nat } 0}$, and characteristic magnetic island widths, $W_{\mathrm{C}, \mathrm{BS}}$. A stabilizing effect of rotation takes place due to reduction of the seed island width under increase of rotation frequency. For most dangerous modes with $m=2, n=1$ and $m=3, n=2$ the instability depends on the choice of $W_{\mathrm{C}, \mathrm{BS}}$. Modes with higher $m$ and $n$ were found to be stable. In particular, there is no NTM generation for the $m=6, n=4$ and the $m=8, n=4$ modes in whole ranges of considered RMP amplitudes, even exceeding those expected from the ELM control coils, and rotation frequencies, including $\Omega_{\text {nat } 0}=0$.

The example of simulation of the $m=3, n=2$ and $m=2, n=1$ mode dynamics with artificially prescribed evolution of the RMP amplitude is presented in Fig. 7. In this simulation $W_{\mathrm{C}, \mathrm{BS}}=1 \mathrm{~cm}$ was set to maximize the neoclassical drive of the modes. In the process of the helical field penetration, the external RMP generates nonrotating small magnetic islands. It is followed by a significant rise of the magnetic island width due to the development of NTM. Initially this rise of $W$ is accompanied by a beginning of the island non-regular rotation. The further growth of the island results in the mode locking due to the effect of RMP and eddy currents in the vacuum vessel walls on the resonant plasma layer rotation. To identify the specific effects of RMP and NTM in the process of the mode development, the calculations with artificial instant shutdown of the corresponding

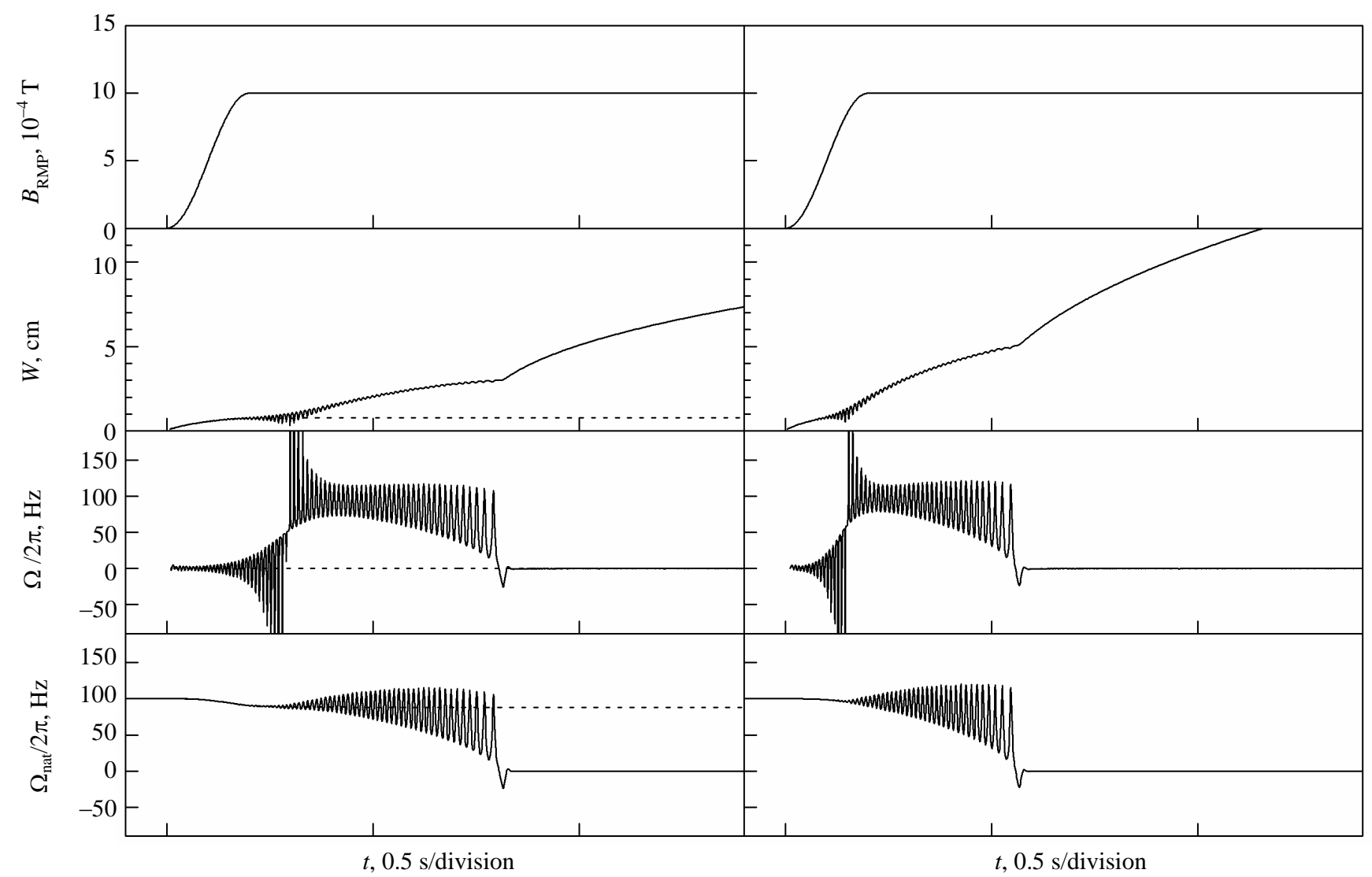

Fig. 7. The development of $m=3, n=2$ (left panel) and $m=2, n=1$ (right panel) modes with the NTM effect (solid curves). The development of $m=3, n=2$ mode without NTM is shown with the dashed curves 
terms in the model were performed. The results of these calculations are presented in Fig. 8. In this figure, one can see that after termination of RMP, the magnetic island continue its development but no locking takes place. In the case of instant shutdown of the NTM terms in equations (17), (18) the magnetic island width reduces to the original seed island level.

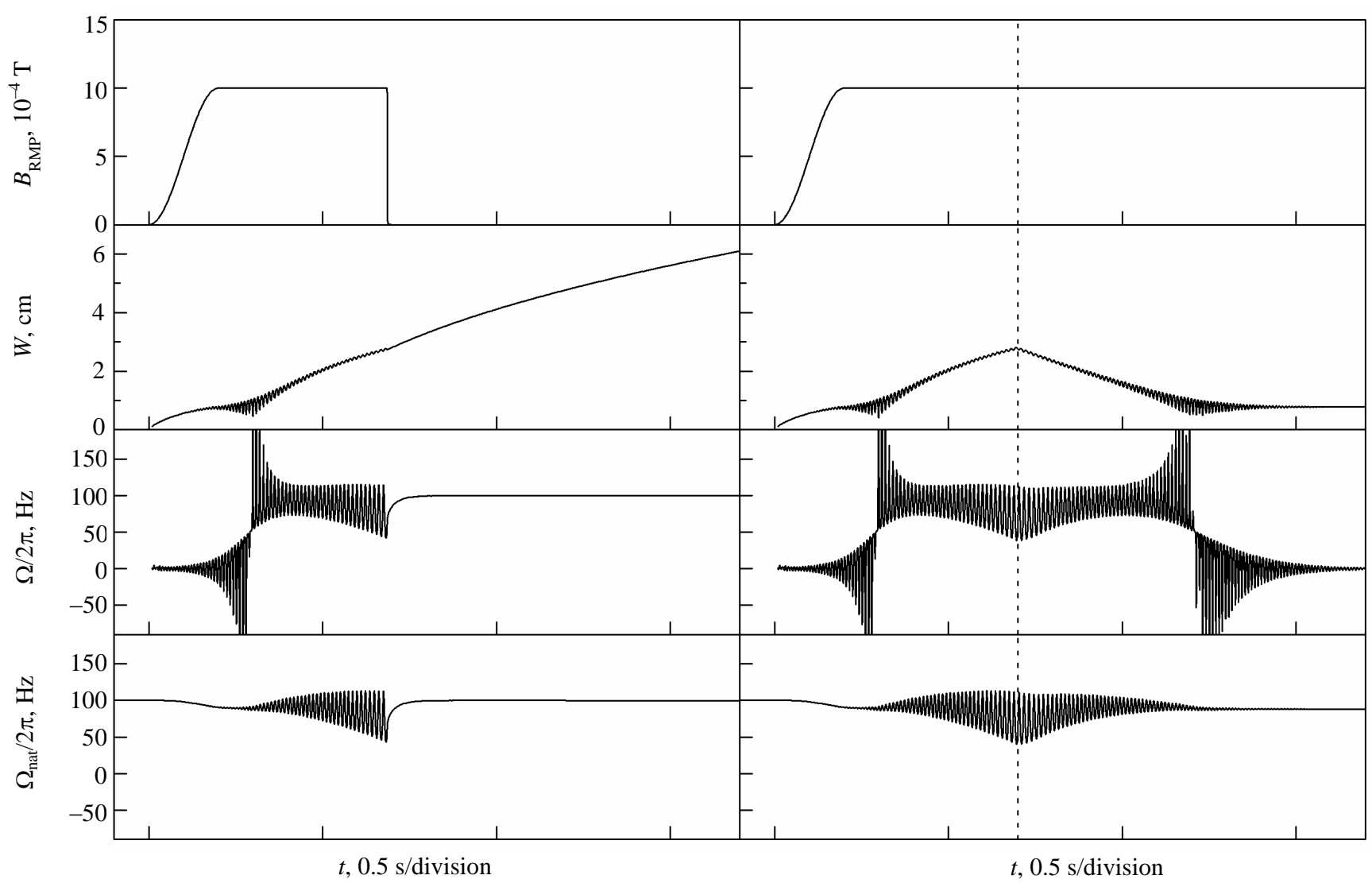

Fig. 8. The $m=3, n=2$ mode development in cases of artificial instant shutdown of RMP (left panel) and NTM term (right panel). The NTM shutdown moment of time is shown with vertical dashed line

\section{SUMMARY}

Dynamics of the magnetic islands caused by the RMP in rotating ITER-like plasma was numerically investigated. The widths of the islands were shown to depend on the RMP amplitudes as well as on the intrinsic plasma rotation frequency. The rotation has a strong stabilizing effect. For the $m=3, n=2$ and $m=2, n=1$ modes, the RMP generated islands can play the role of seed islands for the NTM development. The RMP caused by the ELM control coils were found not to generate the NTM at the $q=3 / 2$ and $q=2$ magnetic surfaces. In future, we plan to carry out the TEAR code benchmarking against other MHD codes and its experimental validation as well as further development of the code to take into account the toroidal effects on the tearing modes dynamics.

\section{ACKNOWLEDGEMENTS}

We thank P.B. Aleynikov, Yu.V. Gribov, V.D. Pustovitov for helpful discussions and assistance. This work is supported by the Rosatom State Nuclear Energy Corporation.

\section{REFERENCES}

1. Ivanov N.V., Kakurin A.M., Konovalov S.V. Magnetic island dynamics under external magnetic perturbation in rotating resistive tokamak Plasma. - In: Proc. 24th IAEA Fusion Energy Conf. San-Diego, USA, 2012, IAEA-CN-197/TH/P3-22: http://www-naweb.iaea.org/napc/physics/FEC/FEC2012/papers/342_THP322.pdf.

2. Chudnovskiy A.N., Gvozdkov Yu.V., Ivanov N.V., Kakurin A.M. et al. MHD-mode locking by controlled halocurrent in the T-10 tokamak. — Nucl. Fusion, 2003, vol. 43, pp. 681—685. 
3. Ivanov N.V., Kakurin A.M. Locking of small magnetic islands by error field in T-10 tokamak. - Problems of Atomic Science and Technology. Series Thermonuclear Fusion, 2012, issue 1, pp. 64-71, http://vant.iterru.ru/engvant_2012_1/6.pdf http://elibrary.ru/download/86673257.pdf

4. Rutherford P.H. Nonlinear growth of the tearing mode. — Phys. Fluids, 1973, vol. 16, pp. 1903 - 1908.

5. Monticello D.A., White R.B. Nonlinear drift tearing modes. — Phys. Fluids, 1980, vol. 23, pp. 366-371.

6. Yu Q., Guenter S., Kikuchi Y., Finken K.H. Numerical modelling of error field penetration. — Nucl. Fusion, 2008, vol. 48, p. 024007.

7. Ferraro N.M., Jardin S.C., Snyder P.B. Ideal and resistive edge stability calculations with M3D-C. - Phys. Plasmas, 2010, vol. 17, p. 102508.

8. Braginskii S.I. - Reviews of Plasma Physics. Edited by M. Leontovich (Consultants Bureau, New York.), 1965, vol. 1, pp. $205-311$

9. White R.B. et al. Saturation of the tearing mode. — Phys. Fluids, 1977, vol. 20, pp. 800 -805.

10. Hender T.C. et al. Progress in the ITER Physics Basis. Chapter 3: MHD stability, operational limits and disruptions. - Nucl. Fusion, 2007, vol. 47, pp. S128-S202.

11. Sauter O., La Haye R.J., Chang Z. et al. Beta limits in long-pulse tokamak discharges. — Phys. Plasmas, 1997, vol. 4, pp. $1654-1664$.

12. Mikhailovskii A.B. Theory of magnetic islands in tokamaks with accenting neoclassical tearing modes. — Contrib. Plasma Phys., 2003, vol. 43, pp. 125-177.

13. Fitzpatrick R. Interaction of tearing modes with external structures in cylindrical geometry. — Nucl. Fusion, 1993, vol. 33, pp. 1049-1084.

14. Kikuchi Y., Finken K.H., Jakubowski M. et al. Modelling of the penetration process of externally applied helical magnetic perturbation of the DED on the TEXTOR tokamak. - PPCF, 2006, vol. 48, pp. 169-183.

15. Hosea J.C., Jobes F.C., Hickok R.L., Dellis A.N. Rotation and structure of low-frequency oscillations inside the STTokamak plasma. - Phys. Rev. Letters, 1973, vol. 30, pp. 839-842.

16. Koslowski H.R., Liang Y., Kramer-Flecken A. et al. Dependence of the threshold for perturbation field generated $m / n=2 / 1$ tearing modes on the plasma fluid rotation. — Nucl. Fusion, 2006, vol. 46, pp. L1—L5.

17. Putvinski S.V. ITER IDM UID: 478WJZ, 2011.

18. Gribov Y.V., Pustovitov V.D. Analytical study of RWM feedback stabilisation with application to ITER. - In: Proc. 19th IAEA Fusion Energy Conf. Lyon, France, 2002, IAEA/CN-94/CT/P-12.

19. Tala T. et al. Parametric dependences of momentum pinch and Prandtl number in JET. — Nucl. Fusion, 2011, vol. 51, p. 123002.

20. Gribov Y. ITER IDM UID: 4GKRYC, 2011.

21. Aleynikov P.B. et al. Simulations of runaway electron transport under MHD perturbations in ITER. — In: 37 th EPS Conf. on Plasma Physics. Dublin, Ireland, 2010, P1.1004.

22. Gribov Yu.V. ITER IDM 33NHXN v. 3.9, 2011.

23. Konovalov S.V. et al. Role of anomalous transport in onset and evolution of neoclassical tearing modes. - Plasma Phys. Control. Fusion, 2005, vol. 47, pp. B223-B236.

24. Sauter O., Angioni C., Lin-Liu Y.R. Neoclassical conductivity and bootstrap current formulas for general axisymmetric equilibria and arbitrary collisionality regime. — Phys. Plasmas, 1999, vol. 6, pp. 2834-2839.

25. Mikhailovskii A.B., Konovalov S.V., Shirokov M.S., Tsypin V.S. Effect of the magnetic field curvature on magnetic islands in tokamaks. — Plasma Phys. Rep., 2004, vol. 30, pp. 549—567.

\section{AUTHORS}

Ivanov N.V. NRC “Kurchatov Institute”, pl. Kurchatova 1, 123182 Moscow, Russia; Ivanov_NV@nrcki.ru

Kakurin A.M. NRC “Kurchatov Institute”, pl. Kurchatova 1, 123182 Moscow, Russia

Konovalov S.V NRC “Kurchatov Institute”, pl. Kurchatova 1, 123182 Moscow, Russia; Konovalov_SV@nrcki.ru 